\title{
CrMPK3, a mitogen activated protein kinase from Catharanthus roseus and its possible role in stress induced biosynthesis of monoterpenoid indole alkaloids
}

Susheel Kumar Raina, Dhammaprakash Pandhari Wankhede, Monika Jaggi, Pallavi Singh, Siddhi Kashinath Jalmi, Badmi Raghuram, Arsheed Hussain Sheikh and Alok Krishna Sinha*

\begin{abstract}
Background: Mitogen activated protein kinase (MAPK) cascade is an important signaling cascade that operates in stress signal transduction in plants. The biologically active monoterpenoid indole alkaloids (MIA) produced in Catharanthus roseus are known to be induced under several abiotic stress conditions such as wounding, UV-B etc. However involvement of any signaling component in the accumulation of MIAs remains poorly investigated so far. Here we report isolation of a novel abiotic stress inducible Catharanthus roseus MAPK, CrMPK3 that may have role in accumulation of MIAs in response to abiotic stress.

Results: CrMPK3 expressed in bacterial system is an active kinase as it showed auto-phosphorylation and phosphorylation of Myelin Basic Protein. CrMPK3 though localized in cytoplasm, moves to nucleus upon wounding. Wounding, UV treatment and MeJA application on C. roseus leaves resulted in the transcript accumulation of CrMPK3 as well as activation of MAPK in C. roseus leaves. Immuno-precipitation followed by immunoblot analysis revealed that wounding, UV treatment and methyl jasmonate (MeJA) activate CrMPK3. Transient over-expression of CrMPK3 in C. roseus leaf tissue showed enhanced expression of key MIA biosynthesis pathway genes and also accumulation of specific MIAs.
\end{abstract}

Conclusion: Results from our study suggest a possible involvement of CrMPK3 in abiotic stress signal transduction towards regulation of transcripts of key MIA biosynthetic pathway genes, regulators and accumulation of major MIAs.

Keywords: Catharanthus roseus, Methyl jasmonate, Mitogen activated protein kinase, Monoterpenoid indole alkaloid, Secondary metabolism

\section{Background}

Plants in order to cope-up with changing environmental conditions synthesize a wide variety of secondary metabolites, which not only acts as defence molecules but also contribute to its overall growth and development. Biosynthesis of these compounds is often induced by various environmental stimuli and stress factors such as UV light or pathogen attack. Catharanthus roseus (L.) G. Don, a tropical plant species, synthesizes more than 130

\footnotetext{
*Correspondence: alok@nipgr.ac.in

National Institute of Plant Genome Research (NIPGR), Aruna Asaf Ali Road, New Delhi 110067, India
}

monoterpenoid indole alkaloids (MIAs) as part of its secondary metabolism. Some of the MIAs possess high therapeutic value such as antineoplastic drugs vinblastine and vincristine, so have earned a great commercial importance [1]. These MIAs are produced at very low levels via a complex MIA biosynthetic pathway that is also reported to be stress induced in C. roseus. However, factors such as fungal elicitors, heavy metal ions, UV radiation, osmotic shock, wounding or pathogen attack induce their biosynthesis. Treatment of $C$. roseus seedlings with methyl jasmonate (MeJA) increases the activity levels of TDC (tryptophan decarboxylase), STR (Strictosidine synthase), 
D4H (Desacetoxyvindoline -4-hydroxylase) and DAT (Deacetylvindoline 4-O-acetyltransferasee) and leads to enhanced accumulation of vindoline [2]. Moreover, elicitor induced JA biosynthesis and MeJA induced $T d c$ and Str are blocked by K-252a, a protein kinase inhibitor suggesting involvement of protein phosphorylation in this signal transduction [3]. To date there is no information regarding the involvement of any signaling component towards the accumulation of alkaloids in response to stress. One can speculate the involvement of mitogen activated protein kinase (MAPK) cascade since wounding and systemin have been known to activate MAPKs upstream of octadecanoid pathway in tomato plants as well as autotrophic cell cultures [4]. In Arabidopsis, regulation of camalexin biosynthesis by MPK3/MPK6 cascade has been reported [5] MAP kinase is one of the major signaling cascades by which extracellular stimuli are transduced into intracellular responses [6]. MAPK cascade includes three functionally linked kinases: MAP Kinase (MAPK); MAPK kinase (MAPKK) and MAPKK kinase (MAPKKK). Upon elicitation by external stimuli, the receptors mediate the phosphorylation and activation of MAPKKK. This activated MAPKKK activates MAPKK by phoshorylation at serine and threonine residues, which in turn activates MAPK by phosphorylation at threonine and tyrosine residues [7]. MAPKs are known to be activated by a variety of biotic and abiotic [6-9] stresses in plants. In mammals and yeast, MAPK cascades are active downstream to G-protein coupled receptors, receptor tyrosine kinases (RTKs) or two component histidine kinases. Most of the MAPK substrates include transcription factors, transcription regulators, splicing factors, receptors, histones and others [10]. The role of MAPKs has been implicated in various biological phenomena in plants including pathogen defense, abiotic stresses, cytokinesis and cell differentiation, and plant hormone signaling [7].

In the present study we report cloning of a mitogen activated protein kinase from $C$. roseus, CrMPK3. The transcript and activity of CrMPK3 is induced by the same stimuli known to induce alkaloid biosynthesis in C. roseus. Further, transient over-expression of CrMPK3 in $C$. roesus leaves results in up-regulation of specific alkaloids and the transcripts of genes involved in MIA biosynthesis pathway. We infer that CrMPK3 may have its role in stress-mediated accumulation of MIA in C. roseus.

\section{Results}

\section{Methyl jasmonate, UV and wounding activate MAP} kinases in $C$. roseus

MAP kinase cascade is known to regulate several stress responsive biological processes in different plants. In order to understand the signaling component of MIA biosynthesis we checked activation of MAPK in conditions that lead to accumulation of MIAs. In C. roseus methyl jasmonate treatment $[2,11]$ and stress conditions such as UV [12] and wounding [13] have been shown to enhance alkaloid accumulation. We therefore, used these three conditions to study MAPK activity in C. roseus leaves. Equal amounts of protein extracted from the treated leaf tissue were analyzed in an in-gel kinase assay using myelin basic protein (MBP) as an artificial substrate. As shown in Figure 1, a transient activation of two different MAP kinases were observed within 15 minutes of wounding that decreased gradually. UV treatment also leads to increase in kinase activity within 30 minutes of treatment. At least three MBP phosphorylating kinases were observed after UV exposure whereas methyl jasmonate led to activation of single MAPK (Additional file 1: Figure 1).

\section{Cloning of CrMPK3}

Having seen the activation of MAPK in different stress conditions we attempted cloning MAP kinase gene from C. roseus that might be regulating MIA pathway. For the purpose, a cDNA library of $C$. roseus in $\lambda \mathrm{ZAP}$ II was screened by a 438 bp MAPK EST (GenBank accession No. AJ537469). The sequencing of positive plaques obtained after tertiary screening gave a 630 bp fragment that showed maximum identity with Wound Induced Protein Kinase (WIPK) from Nicotiana attenuata (ABJ89813). However, the identified clone was a partial gene with missing 5 ' end. To get full-length MAPK gene, a forward degenerate oligonucleotide was designed from $5^{\prime}$ end of several MAPK sequences from other plant species that showed significantly high identity with the 630 bp fragment obtained from library screening. The reverse primer was designed from the $3^{\prime}$ end of the identified partial MAPK clone. PCR amplification of $C$. roseus cDNA with 5' degenerate and 3' gene specific primers

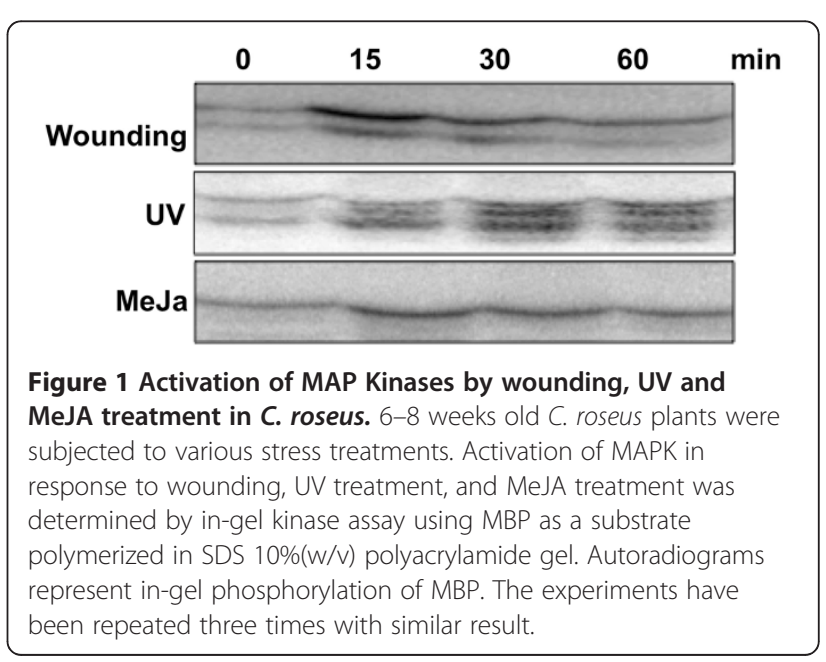


resulted in an amplicon of about $1.1 \mathrm{~Kb}$. The cloning and sequence analysis of the amplicon led to identification of an ORF of 1119 bp long encoding distinct C. roseus MAPK. Since the identified MAPK gene shows maximum identity with tobacco WIPK and Arabidopsis AtMPK3 (Additional file 2: Figure 2) we named it as CrMPK3 (EF156758). Phylogenetic analysis of CrMPK3 (Additional file 2) indicated that it belongs to group A MAPKs [14] that are involved in signaling pathogen infections and abiotic stresses. Analysis of deduced amino acid sequence revealed that the CrMPK3 encodes
372 amino acids and the protein contains all the 11 subdomains (Additional file 3) that are conserved among all MAPK families [15]. The 'TEY' phosphorylation motif is found between VII and VIII subdomains of CrMPK3. In silico analysis of the sequence revealed an approximate molecular weight of $43 \mathrm{kDa}$.

\section{Recombinant CrMPK3 is an active MAPK}

To demonstrate that CrMPK3 encodes a functional MAPK, its complete ORF was cloned into pGEX-4-T2 vector and transformed into protease deficient strain

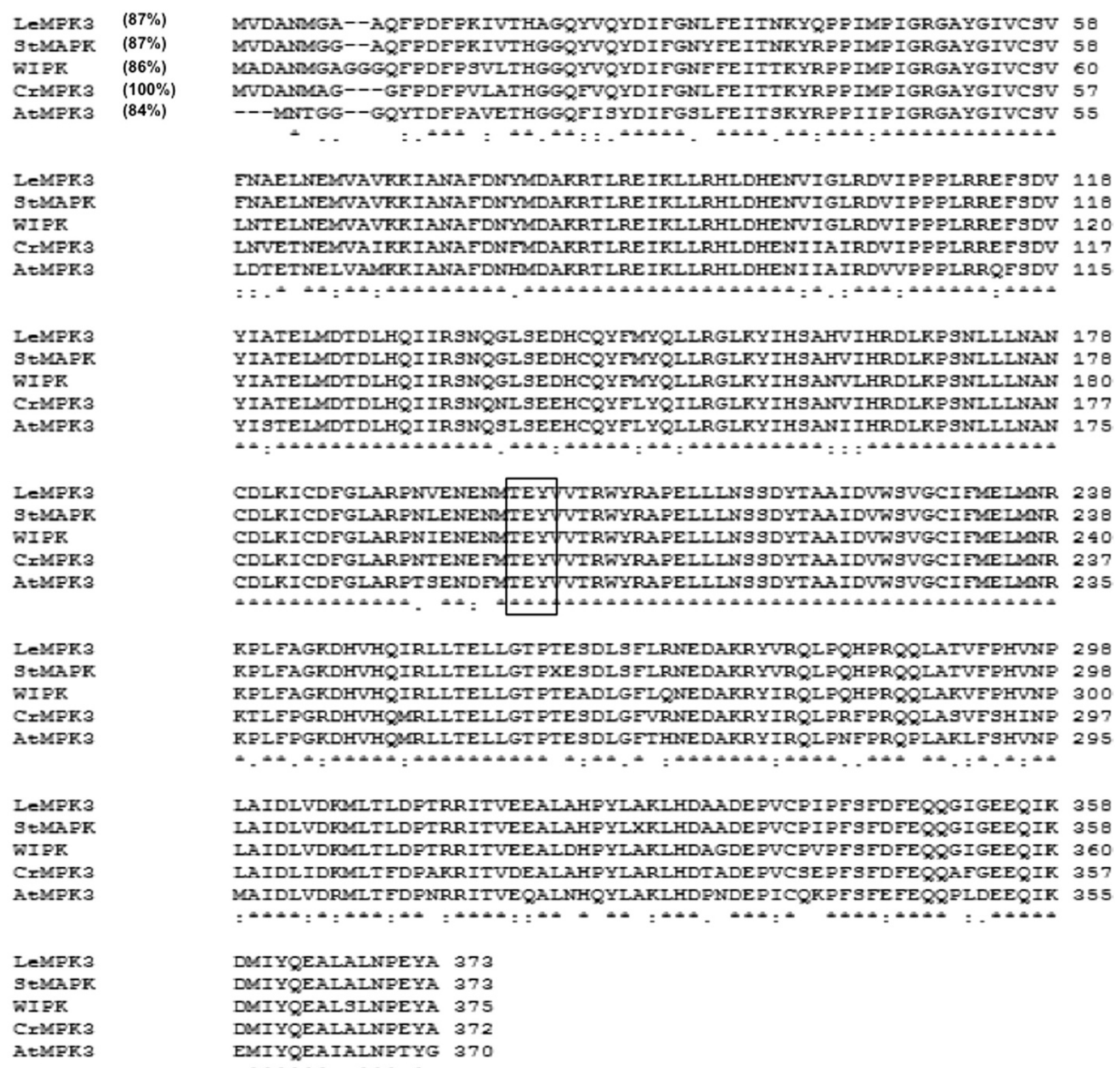

LEMPR3

STMRR ฟI PR

CIMPR3

AtMPR3

LeMPR3

StMARR ลIPR

CIMPR3

AtMPR3

LeMPR3

SEMAPR

WIPR

CIMPR3

At:MPR3

LeMPR3

SEMARK WIPR

CrMPR3

AEMPR3

ENAELWEMVAVRKIAWAFDWMIARRTLREIFLLRHLDHEWVIGLRDVIPPPIRREESDV 118 FWAELWEYVAVRKIAWAFDWYIDARRTLREIFLIRHLDHEWVIGLRDVIPPPIRREFSDV 118 LWTELWEMVAVRTAWAFDWYMARRTLREIFLIRHLDHEWVIGLRDVIPRPIRREFSDV 120 LWVETWEMVIFRIAWAFDWEMDARRTLREIFLIRHLDHENIIAIRDVIPRPIRREFSDV 117 LDTETWELVAYRKIAWAEDWHADARRTLREIKLLRHLDHEWIIAIRDVVPPPIRRQESDV 115

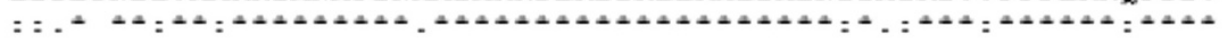

YIATELNDTDLHQIIRSNQGLSEDHCQYEYYQLLRGLKYIHSAHVIHRDLFPSNLILNAN 178 YIATELNDTDLHQIIRSNQGLSEDHCQYEWYQLRGLRYIHSAHVIHRDLRPSNLILNAN 178 YIATELNDTDLHQIIRSNQGLSEDHCQYEYYQLIRGLKYIHSANVLHRDLRPSNLILNAN 180 YIATELMDTDLHQIIRSNQNLSEEHCQYELYQILRGLKYIHSANVIHRDIRPSNLILNAN 177 YISTELNDTDLHQIIRSWQSLSEEHCQYELYQLLRGLKYIHSANIIHRDLRPSWLLINAN 175

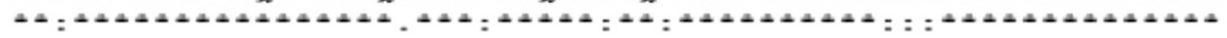

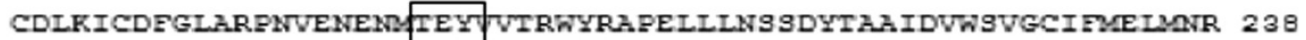
CDLKICDEGLARPNLENENATEY VTRWYRAPELILWSSDYTAAIDVNSVGCIEYEIMATR 238 CDIKICDEGLARPNIENENATEY VTRWYRAPELILNSSDYTAAIDVWSVGCIEMELARR 240

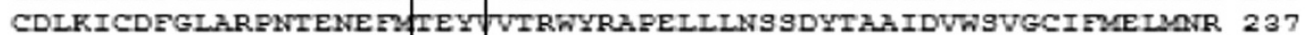
CDIRICDEGLARPTSENDEYTEY VTRWYRAREILINSSDYTAAIDVWSVGCIEMEILAN 235

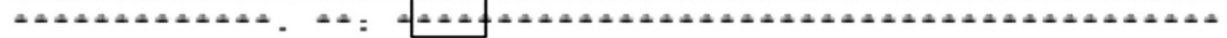

KPLEAGRDHVHQIRLITELLGTPTESDLSELRWEDARRYVRQLPQHPRQQIATVEPHVIP 298 KPLEAGRDHVHQIRLITELLGTPXESDIS ELRTEDARRYVRQLPQHPRQQLATVEPHVNP 298 KPLEAGKDHVHQIRLITELLGTPTEADLGELQNEDAFRYIRQLPQHPRQQLAKVEPHVNP 300 KTIEPGRDHVHQURLITELLGTPTESDIGEVRWEDARRYIRQLPREPRQQLASVESHINP 297 KPIEPGRDHVHQMRILTELIGTPTESDLGETHNEDARRYIRQIPNEPRQPIAFIESHVNP 295

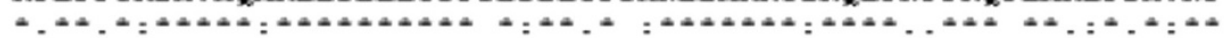

LeMPR3 SEMARK

LAIDIVDFOIIIDPTRRITVEEALAHPYLAKLHDAADEPVCPIPESEDEEQQGIGEEQIK 358 LAIDIVDFOITLDPTRRITVEEALAHPYLXKLHDAADEPVCPIPESEDE'QQGIGEEQIK 358 LAIDIVDFOIILDPIRRITVEEALDHPYLAKLHDAGDEPVCPVPESEDEEQQGIGEEQIK 360 LAIDIIDFOITEDPAFRITVDEALAHPYLARLHDTADEPVCSEPESFDEEQQAEGEEQIK 357 CIMPR3 MAIDIVDRUITEDPNRRITVEQALWHQYLAKLHDPNDEPICQKPESEEEEQQPIDEEQIK 355 2tMPR3

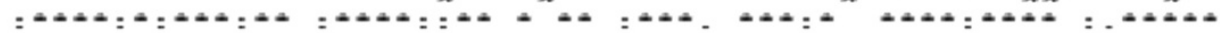

LeMPR3 SEMRPR DUIYQEALALWPEYA 373 DOIYQEALALNPEYA 373 DUIYQEALIITEYA 375 DOIYQEALALNPEYA 372 EMIYQEARINPTYG 370

CIMPR3 2terPR3

Figure 2 Multiple sequence alignment of CrMPK3 and related MAPK sequences using ClustalW(1.82). The NCBI accession numbers are Lycopersicon esculentum LeMPK3 (AY261514), Solanum tuberosum MAP Kinase, (AB206552), Nicotiana attenuata WIPK (DQ991136) and A. thaliana AtMPK3 (NM114433). 
of E. coli BL21. The bacterially expressed glutathione S-transferase (GST):CrMPK3 fusion protein was affinity purified and used in an in-solution kinase assay alone as well as with myelin basic protein (MBP) as substrate. The recombinant protein showed auto-phosphorylation as well as phosphorylation of MBP (Figure 3A) suggesting that $C r M P K 3$ encodes an active MAP kinase. Kinase inactive form of CrMPK3 generated by mutating lysine (K) at 69th amino acid residue to arginine (R) in ATP binding site, CrMPK $3^{\mathrm{K} 69 \mathrm{R}}$ failed either to auto phosphorylate or to phosphorylate MBP (Figure 3A). The CrMPK3 activity was further analyzed in an in-gel kinase assay with decreasing amount of recombinant protein in factor of 10 using MBP as substrate. As expected, the activity of purified fusion protein decreased with decrease in kinase concentration (Figure 3B). The inactive form, CrMPK $3^{\mathrm{K} 69 \mathrm{R}}$ showed no activity in in-gel kinase assay (data not shown).

\section{CrMPK3 moves to nucleus upon wounding}

To understand the fate of CrMPK3 in planta, subcellular localization of CrMPK3 was checked. For this, reading frame of $C r M P K 3$ was fused in frame with that of Green Fluorescent Protein (GFP) in pCAMBIA 1303 vector. Agrobacterium tumefaciens strain GV3101 transformed
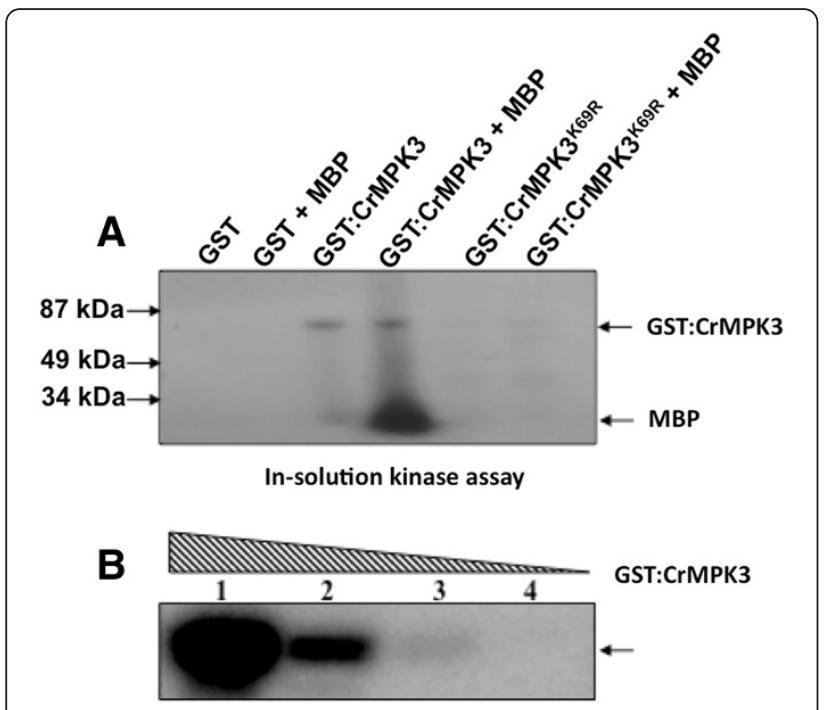

In-gel kinase assay

Figure 3 CrMPK3 shows autophosphorylation as well as MBP phosphorylation. (A) CrMPK3 expressed in E. coli, BL21 strain as GST fusion was analyzed for autophosphorylation, as well as phosphorylation of MBP in an in-solution kinase assay using radiolabled ATP. GST alone was used in the assay and showed no auto- and substrate phosphorylation. (B). An in-gel kinase assay with decreasing concentrations of GST-CrMPK3 using MBP as substrate. The amount of recombinant proteins used was $1 \mu \mathrm{g} ; 0.1 \mu \mathrm{g} ; 0.01 \mu \mathrm{g}$; $0.001 \mu \mathrm{g}$ from lane1 to 4 . The phosphorylated MBP was visualized by autoradiography. The experiments have been repeated three times with similar result. with this construct was used to transiently transform C. roseus leaf discs and viewed under confocal microscope after three days of incubation. CrMPK3 was found localized mostly inside the cell along plasma membrane and cytoplasm (Figure 4). Plasmolysis of the cells using mannitol have confirmed the fusion protein to be localized in plasma membrane and cytoplasm (Additional file 4). However, when the leaf tissue was wounded by cutting into smaller pieces and viewed under microscope after 5 min of wounding, there appeared a shift in GFP fluorescence dispersed mostly in cytoplasm (Figure 4). When the same slide was observed after ten minutes, most of the fluorescence was found localized to nucleus (Figure 4). Positions of the nucleus in slide are shown by DAPI staining which coincides to that of CrMPK3 nuclear signal (Additional file 5). However, neither the CrMPK $3^{\mathrm{K} 69 \mathrm{R}}$-GFP nor GFP alone showed wounding induced migration to nucleus (Figure 4). This indicates that only the active CrMPK3 migrates to nuclei upon activation by wounding. This finding is consistent with earlier reports of translocation of MAPK from cytoplasm to nucleus in response to external stimuli [16].

\section{CrMPK3 shows wounding, UV and MeJA inducible expression and activity}

Since tobacco WIPK and Arabidopsis MPK3, the close orthologs of CrMPK3 exhibit increase in both enzymatic activity and mRNA levels in response to various stimuli [17-19] we investigated whether CrMPK3 gene is also induced by stress conditions that enhance MIA accumulation. Two-months-old in vitro grown C. roseus plants were subjected to different stress conditions like wounding and UV treatment as well as methyl jasmonate treatment. The transcript level of CrMPK3 in response to these stresses was analyzed by northern blot analysis. A significant increase in the level of CrMPK3 transcript was noted within 30 minutes of these treatments (Figure 5A). In case of wounding and MeJA treatments, the transcript level started to decline after $30 \mathrm{~min}$ of treatment. However, under UV treatment higher level of the CrMPK3 transcript could be observed up to 2 hours post treatment before declining back to the basal level.

To check whether stress inducible transcript accumulation of CrMPK3 corroborates with its activity in planta, an immunoblot assay was performed using anti-CrMPK3 antibody. Leaves were subjected to wounding, UV stress treatment and methyl jasmonate application, the protein extracts prepared 15 minutes post treatments were immunoprecipitated with antiphosphotyrosine antibody $4 \mathrm{G10}$. The immunoprecipitates were subjected to western blot analysis with anti-CrMPK3 antibody. A distinct activation of $43 \mathrm{kDa}$ MAP kinase is recognized by anti-CrMPK3 antibody in wounding, 


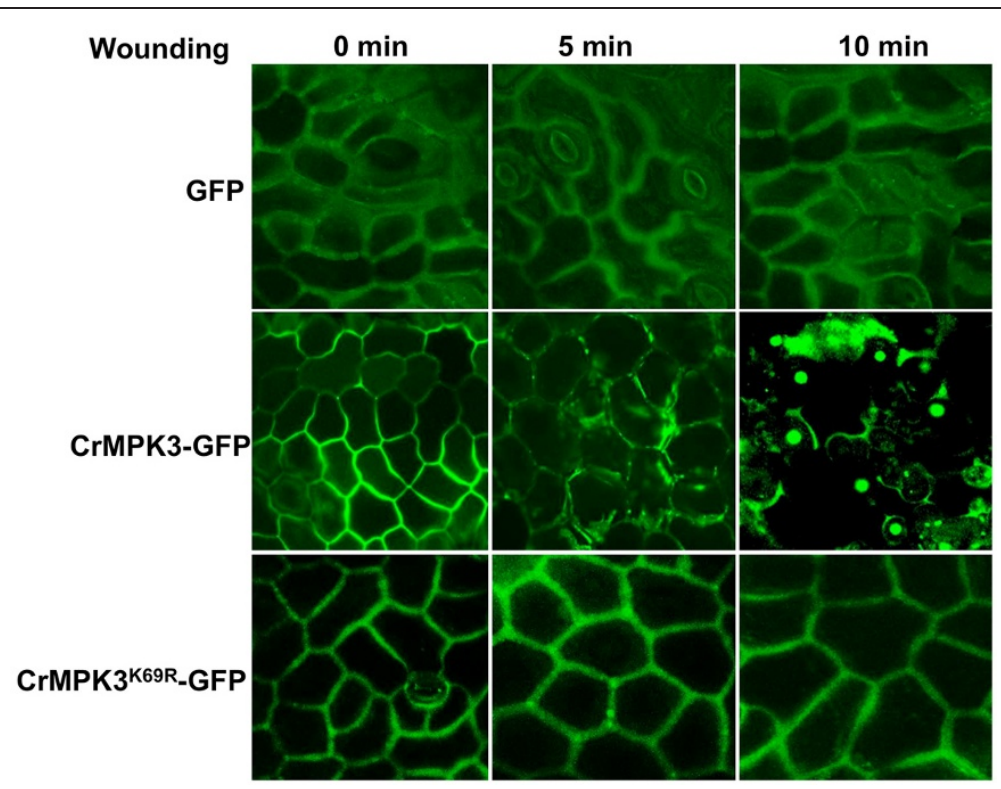

Figure 4 CrMPK3 migrates towards nucleus upon wounding. C. roseus leaf discs transiently transformed with CrMPK3-GFP/CrMPK3 ${ }^{\mathrm{K} 69 R}$ in pCAMBIA1303 vector or only pCAMBIA1303 vector and observed under confocal microscope without wounding and after wounding at the mentioned time points. The experiment was repeated twice with similar results.

UV and MeJA treated tissues compared with control/ mock treated sample (Figure 5B).

Since CrMPK3 and AtMPK3 show high sequence similarity, the commercially available AtMPK3 antibody (Sigma-aldrich) were used to assess activity of CrMPK3 in response to the stress conditions mentioned above in C. roseus leaves. The immunoprecipitation with the dually phosphorylated active form of the MAPK ERK1 (anti-pTEpY) antibody and immunoblot with $\alpha$ AtMPK3 showed the near similar results as were seen with CrMPK3 antibody (Additional file 6). Additionally, when immunoblot was perforemed using $\alpha \mathrm{CrMPK} 3$ against total protein extracts of Atmpk3 mutnat line and wild type plants ( $\mathrm{Col} 0$ ), the signals could be observed only in wild type plants (Additional file 7) validating the specificity of $\alpha$ CrMPK3 antibody.

Transcript up regulation and protein activation of CrMPK3 by wounding, UV treatment and methyl jasmonate prompted us to study key MIA pathway genes regulation by these stresses. C. roseus leaf tissue subjected to wounding, UV treatment and methyl jasmonate application was harvested at different time intervals post treatment. RNA was extracted from these tissues and quantitative-real time-PCR (qRTPCR) was carried out for different genes of MIA pathway. The MIA genes analyzed were Str (Strictosidine synthase), D4h (Desacetoxyvindoline -4-hydroxylase), Dat (Deacetylvindoline 4-O-acetyltransferase) and Tdc (tryptophan decarboxylase) [20]. As shown in Figure 5C, all the treatments resulted in increase in expression of all the four MIA pathway genes. However only a slight increase in expression of Str is observed upon UV treatment.

\section{Transient over-expression of CrMPK3 in C. roseus leaves increases MIA pathway gene expression and alkaloid accumulation}

The mRNA expression pattern of CrMPK3 and key MIA pathway genes has shown a distinct co-regulation in response to different stress treatments. To draw a causal relationship between $C r M P K 3$ and MIA pathway genes and specific alkaloids, transient over-expression of CrMPK3 and its kinase inactive version $C r M P K 3^{K 69 R}$ in $C$. roseus leaves were used. First we confirmed the presence of transgene in transiently transformed $C$. roseus leaves using genomic DNA PCR (Figure 6A). Expression analysis showed enhanced level of CrMPK3 expression in $C r M P K 3, C r M P K 3^{K 69 R}$ transformed leaves than that of control leaves (vector transformed) (Figure 6A, B). We then checked the expression of MIA pathway genes (Tdc, Str, D4h, Dat) and positive regulators ORCA3 (Octadecanoid Responsive cathranthus APII domain factor 3) and repressors $Z C T 1,2,3$ (Z box binding factor 1 , $2,3)$ in the same samples using qRT-PCR analysis. The result obtained revealed a distinct up-regulation in the expression of Tdc, Str, D4h, Dat and Orca3 and down regulation of Zct1, Zct2 and Zct3 in CrMPK3 but not in $\mathrm{CrMPK}^{\mathrm{K} 69 \mathrm{R}}$ transformed leaves (Figure $6 \mathrm{~B}$ ) compared to the empty vector transformed control leaves. In case of $C r M P K 3^{K 69 R}$ transformed leaves, inspite of high level of CrMPK3 mRNA transcripts almost on par with 


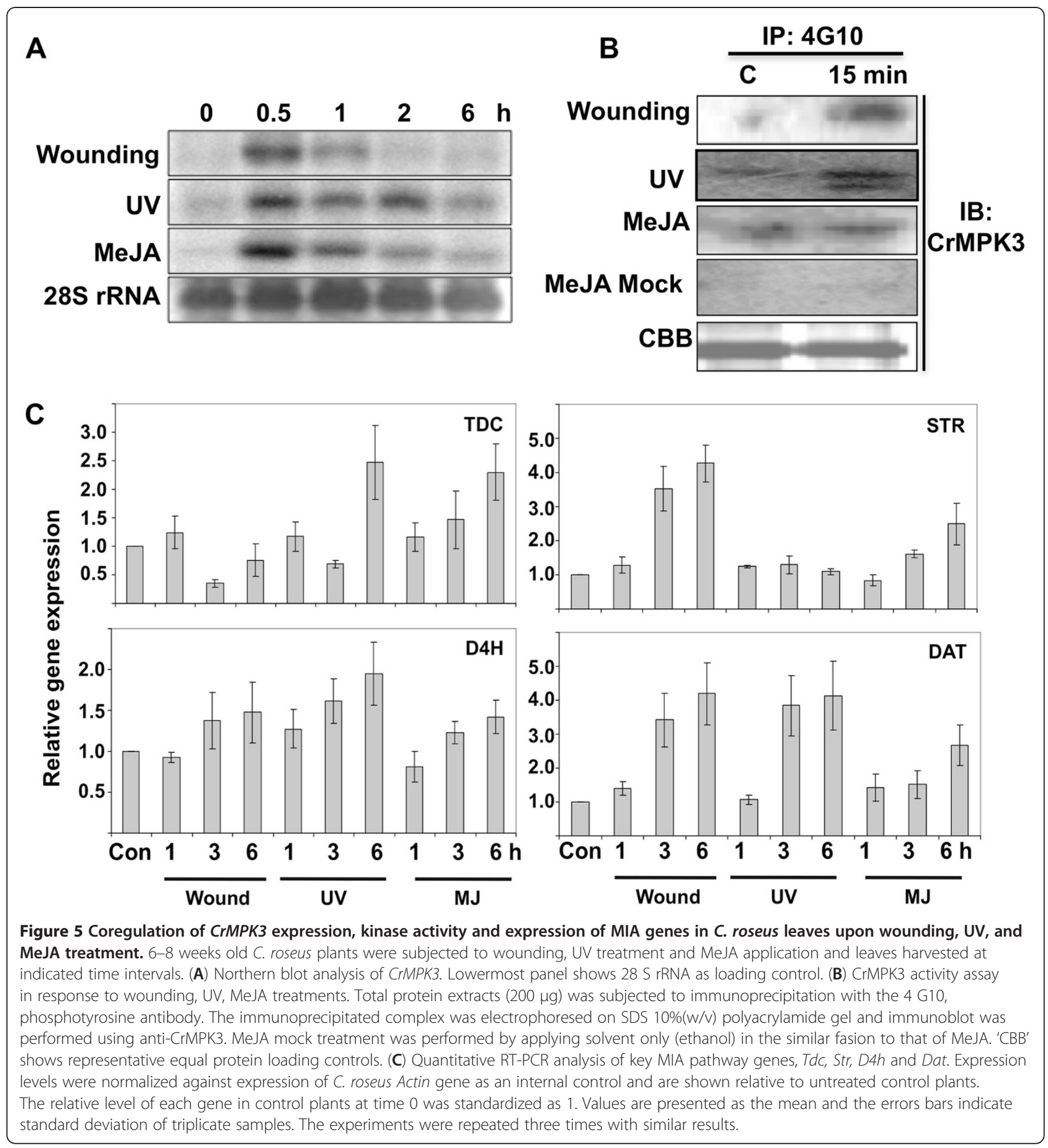

CrMPK3, there were no major changes in transcripts level of MIA biosynthetic pathway genes (Figure 6B). The data obtained indicated that CrMPK3 is involved directly or indirectly in the up-regulation of MIA pathway genes and probably in more accumulation of the MIAs.

To get further insight into the role of CrMPK3 in stress regulated MIA accumulation we quantified some of the specific alkaloids in the transiently transformed
C. roseus leaves. At least 8-10 leaves were used to quantify the alkaloids in both empty vector control and CrMPK3 transformed leaves. The estimation was carried out 72 hours post vacuum infiltration. Interestingly, the accumulation of serpentine, vincristine, vindoline and catharanthine were found to be more in the CrMPK3 transformed leaves (Figure 7). This result further supported the role of CrMPK3 in accumulation of MIAs. 

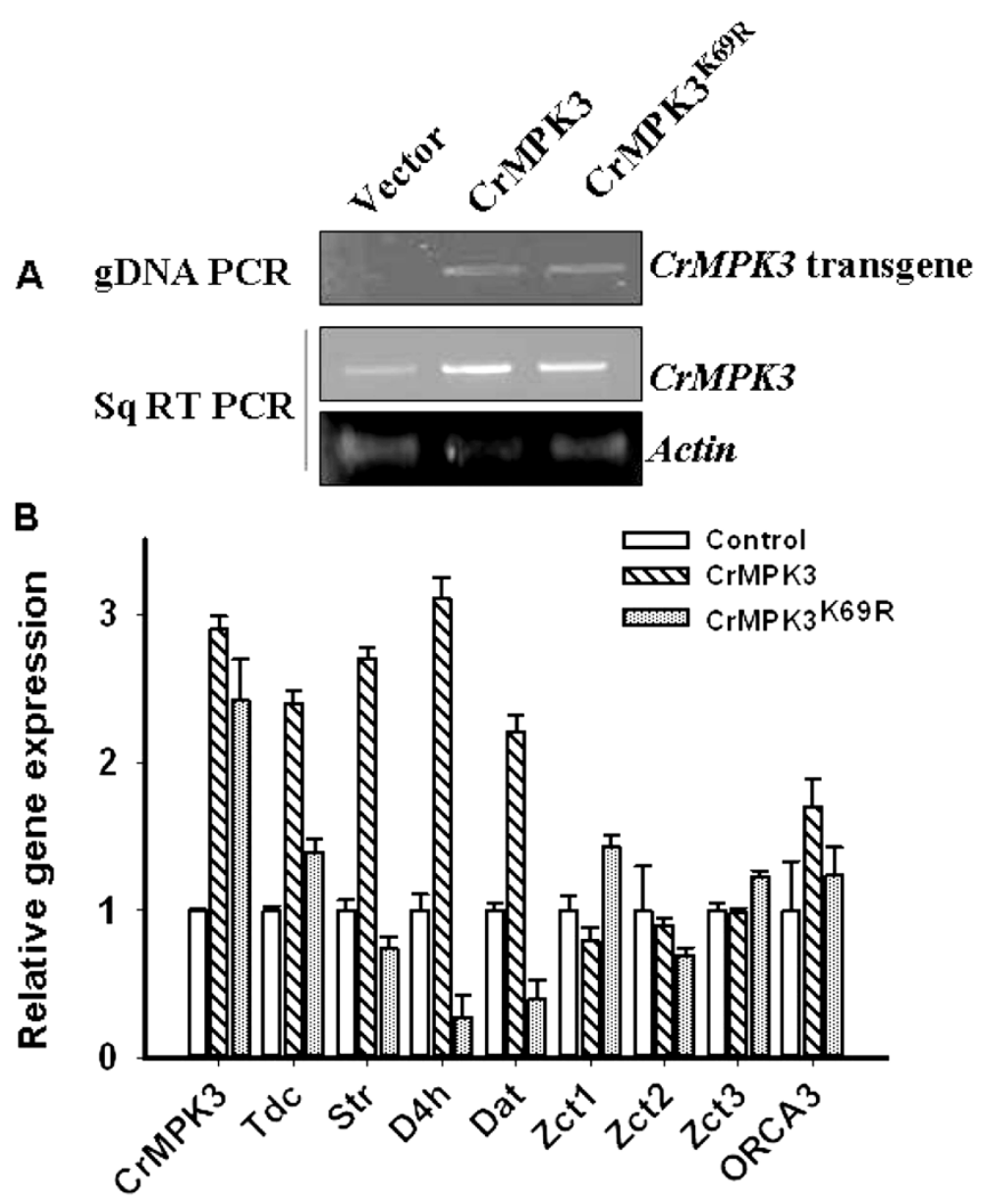

Figure 6 Transient transformation of CrMPK3 in C. roseus leaves increases expression of MIA pathway genes. C. roseus leaves were transiently transformed with pCAMBIA 1303-CrMPK3/CrMPK3 ${ }^{K 69 R}$ construct or pCAMBIA 1303 (Empty Vector) alone by A. tumifaciens mediated transformation. Leaf samples were harvested $72 \mathrm{~h}$ post infiltration for RNA and protein extraction. A- PCR using genomic DNA of CrMPK3/ $C r M P K^{3 K 69 R}$ construct and vector transformed leaves (upper panel). A combination of vector and gene specific primers was used to check the presence of transgene. Middle panel shows expression of CrMPK3 in CrMPK3/CrMPK3 ${ }^{K 69 R}$ construct and vector transformed leaves by sqRT-PCR using gene specific primer pairs. Lower panel shows expressionof Actin gene as equal cDNA loading control. (B) Effect of CrMPK3/CrMPK3 ${ }^{K 69 R}$ transient transformation on transcripts accumulation of key MIA pathway genes and regulators. QRT-PCR analysis was performed to check expression of CrMPK3, Tdc, Str, D4H, Dat and Zct1, Zct2, Zct3 and Orca3. Expression levels were normalized against expression of C. roseus Actin gene as an internal control and are shown relative to empty vector transformed leaf. The relative level of each gene in empty vector transformed leaf sample was standardized as 1 . Values are presented as the mean and the errors bars indicate standard deviation of triplicate samples. The experiments were repeated three times with similar results.

\section{Discussion}

Catharanthus roseus is one of the most pharmaceutically important plant and several efforts have been made to increase the alkaloid content of the plant. Some of them include, use of stress treatments such as wounding, UV treatment, heavy metal elicitation or elicitation by fungal cell wall components or treatment with hormones such as methyl jasmonate. These treatments lead to the increase in expression of MIA pathway genes and accumulation of alkaloids in higher amounts. JA responsive Str and $T d c$ expression is sensitive to protein kinase inhibitors [3] suggesting that phosphorylation plays a key role in the activation of MIA pathway genes. MAP kinases, being the important class of protein kinases involved in signal transduction are operative during biotic or abiotic stresses; its involvement in the elicitor mediated signal transduction leading to higher accumulation of alkaloids cannot be overruled. In this study, we report the isolation of a full-length mitogen activated protein kinase from $C$. roseus, CrMPK3. The deduced amino acid sequence showed $86 \%$ identity with WIPK from Nicotiana benthamiana and LeMPK3 from Lycopersicon esculentum, 84\% identity with AtMPK3 and $78 \%$ identity with OsMPK6. Amino acid sequence analysis revealed the presence of 11 subdomains and a TEY motif between VII and VIII subdomains, typical of a 

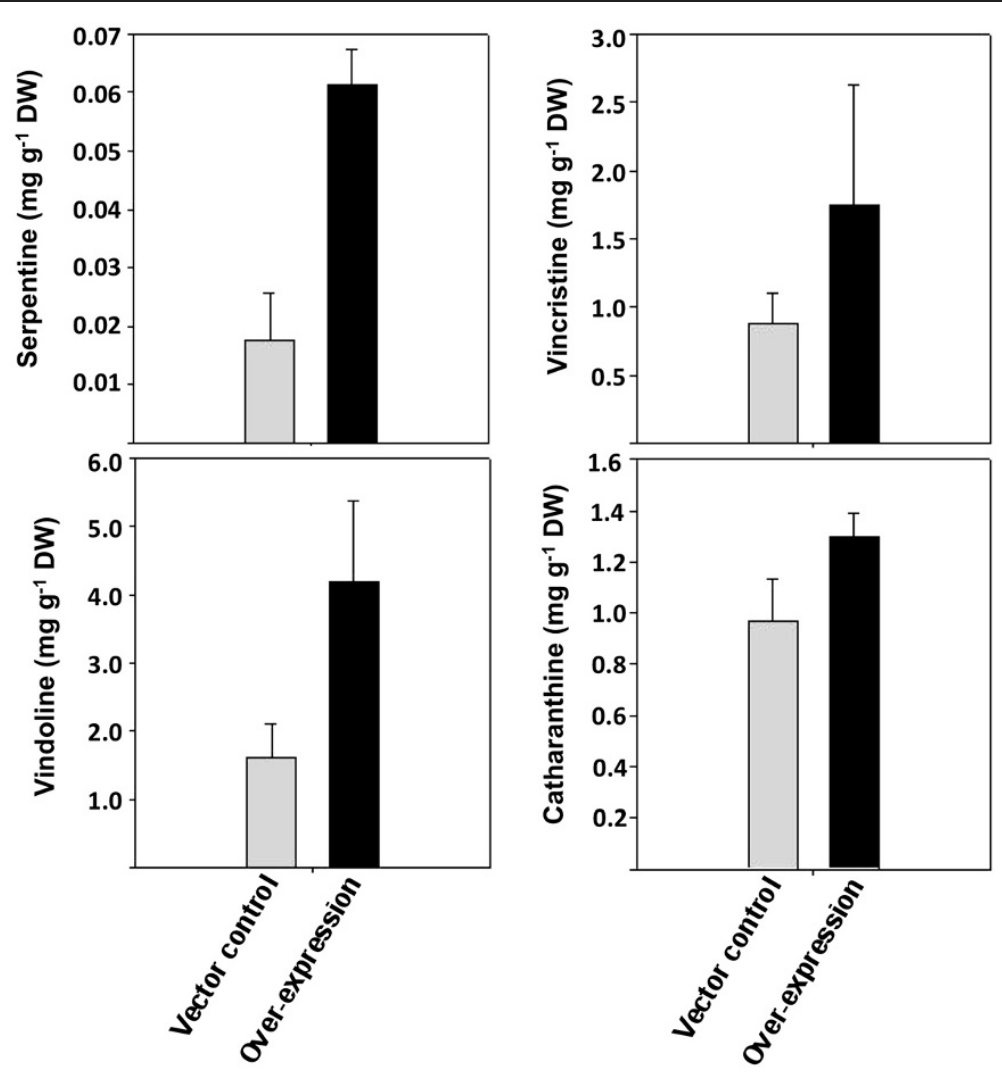

Figure 7 Transient transformation of CrMPK3 in C. roseus leaves increases accumulation of specific MIAs. Specific MIAs, serpentine, vindoline, vincristine and catharanthine accumulation in vector control or CrMPK3 overexpressed leaves were measured. Values are presented as the mean and standard deviation of triplicate samples.

MAP kinase. Further, in solution kinase assay of the GSTtagged CrMPK3 confirmed it to be an active MAP kinase.

Regulations of MAP kinases have been observed both at transcriptional level as well as posttranslational level $[21,22]$. Our findings with CrMPK3 have shown similar results. Enhanced transcript accumulation of $\mathrm{CrMPK3}$ was observed in response to wounding, UV exposure and MeJA treatments. The stress inducible expression of CrMPK3 is in agreement with its orthologs in Arabidopsis (AtMPK3) and other plants where rapid expression was observed for MPK3 in response to stress [23,24]. Further in tomato, UV B specific activation of LeMPK3 has been reported [18]. It is important to note that CrMPK3 showed high homology with LeMPK3. These studies indicate conserved biological function of the close orthologs.

Further, wounding, UV and MeJA activated MAP kinases could be immunoprecipitated with 4 G10, a monoclonal anti-phosphotyrosine antibody [25,26] and identified with CrMPK3 antibody in an immunoblot analysis. The results confirmed that CrMPK3 is activated by wounding, UV and MeJA treatment. Interestingly, an activation of a putative MAPK has been shown in response to $\mathrm{UV}$ in $C$. roseus cell suspension culture
(CSC) [27]. The UV-B responsive putative MAPK activity was found to play significant roles in stimulation of Tdc and Str genes and accumulation of catharanthine in response to UV. However, this specific MAPK has not been identified and gene encoding the same has not been cloned. Nevertheless, the MAPK activity shown earlier [27] is unlikely to be of CrMPK3 since its activity is observed at around $49 \mathrm{kDa}$ where as that of CrMPK3 is of $43 \mathrm{kDa}$. Intriguingly our in gel kinase assay showed activation of more than one MAPK in response to UV. It is important to note that the study [27] has been conducted using $C$. roseus cell suspension culture (CSC) which lack cellular differentiation and provide an entirely different environmental conditions than naturally grown plants. Cellular differentiation has been considered one of the important factors for expression of genes of MIA and its accumulations [28-30]. The difference in activation of distinct MAPKs could be attributed to the different type of biological systems used for these studies. In agreement with this, distinct results with regard to CSC and mature plants have been observed in rice [31].

MAP kinase translocation into nucleus upon their activation is reported in many studies. The nuclear 
translocation of ERM (Elicitor responsive MAP) kinase in parsley within 10 minutes in response to elicitor treatment has been reported [16]. The nuclear translocation of CrMPK3 in response to wounding in C. roseus is in agreement with earlier report [16]. MAP kinases are widely speculated to directly or indirectly interact with transcription factors in the nucleus for gene regulation. The transfer of activated MAP kinase upon wounding to nucleus in the present study supports the function of MAPKs.

Since biosynthesis of MIAs is responsive to environmental signals, transcript accumulation of a few MIA pathway genes in response to wounding, UV treatment and Methyl jasmonate application was studied. A distinct regulation of the MIA pathway genes was observed at transcript level. Since CrMPK3 transcript also showed regulation in response to these treatments, it was thought worthwhile to study the effect of transient overexpression of CrMPK3 on MIA pathway genes. C. roseus leaves were transiently transformed with $C r M P K 3$ binary construct by vacuum infiltration method and transiently transformed leaves were studied for the expression of MIA pathway genes. An increase in CrMPK3 activity was observed in the transiently transformed leaves. The transient transformed leaves also showed a distinct up-regulation in transcription of Tdc, Dat and D4h. Transcript regulation of the positive and negative regulators of MIA pathway genes was also studied. Interestingly, Orca3, a positive regulator of the pathway showed up-regulation, while Zct1, Zct2 and Zct3 the repressors of MIA pathway [32,33], showed down regulation. Zcts are members of zinc finger type transcription factor family and are known repressor of MIA biosynthesis pathway genes [32]. Orca3 is a member of AP2 transcription factor and are known positive regulator of MIA pathway genes [33]. Moreover, the increased transcripts of MIA genes also resulted in higher accumulation of specific alkaloids in transformed leaf samples. A positive correlation has been reported between upregulation of Dat and D4h transcripts and accumulation of related MIAs in C. roseus [34]. The up-regulation in the transcript level of Tdc, Str, Dat and D4h can be partly explained by upregulation of the positive regulator and down-regulation of the repressors. The enhanced transcripts level of key genes of MIA as well as increased accumulation of the specific alkaloids in transiently transformed leaves suggest a positive effect of CrMPK3 on MIA biosynthesis. Role of the members of MAPKs in regulating defence compounds such as phytoalexins have been well documented in Arabidopsis and rice. In Arabidopsis MPK3 and MPK6 have been shown to regulate multiple genes in ethylene and camalexin biosynthesis [5,35]. Similarly in rice MKK4-MPK3/MPK6 cascade regulate expression of several genes of phytoalexin biosynthesis and accumulation of phytoalexins in response to MAMP [36].

Our present study reports, a MAP kinase from a medicinally important, $C$. roseus and its involvement, directly or indirectly in regulating the synthesis of monoterpenoid indole alkaloids. Based on these findings it appears that CrMPK3 could be a potential candidate that can be engineered for better production of specific alkaloid in $C$. roseus.

\section{Conclusions}

In the present study, cloning of the first MAPK, CrMPK3 from medicinally important plant Catharanthus roseus has been reported. The CrMPK3 encodes an active MAP kinase with 372 amino acids and molecular weight $\sim 43 \mathrm{kDa}$. In-silico analysis of amino acid sequence of CrMPK3 shows that it possesses all the basic features of MAP kinase with 'TEY' phosphorylation motif. Phylogenetic analysis of CrMPK3 with closely related MAPKs suggests that it belongs to group A MAPK [14] as it shows high sequence identity with tobacco WIPK and Arabidopsis AtMPK3. Interestingly, CrMPK3 shows UV, wounding and MeJA inducible expression pattern that is in harmony with the expression pattern of many of the key genes and regulators of MIA biosynthetic pathway and MIA accumulations (Figure 5) [33,37]. Enhanced expression of CrMPK3 in the above conditions also reflected in its increased kinase activity in all studied conditions. Role of CrMPK3 in wounding is further substantiated with wounding induced nuclear localization of CrMPK3-GFP, which is otherwise localized in cytoplasm (Figure 4). Further concordance of CrMPK3 and MIAs comes from the transient expression of CrMPK3 in C. roseus leaves, which not only resulted in increased expression of master regulator, Orca3 and key genes of MIA pathway but also more accumulation of important alkaloids such as serpentine, vindoline, vincristine and catharanthine (Figure 6, 7). From these studies we infer that CrMPK3 may form an important component of signal transduction pathway leading to stress induced accumulation of MIAs in C. roseus. Future studies with stable transgenics of CrMPK3 and identification of interacting partner of CrMPK3 in the form of the already identified MIA pathway regulators could shed more light on signal transduction network. Nonetheless, the present work is a miniature step towards unraveling the complex signal transduction mechanism in this model medicinal plant.

\section{Methods}

\section{Plant material and treatments}

Two months old Catharanthus roseus var. Nirmal plants growing in greenhouse at a temperature of $28^{\circ} \mathrm{C}$ were subjected to various stress treatments. For wounding 
intact leaves of $C$. roseus plants were damaged upto $40 \%$ of their leaf lamina using a surgical blade. The leaf samples were harvested at different time points mentioned in the legend of respective figures by snap freezing in liquid $\mathrm{N}_{2}$ and stored in $-80^{\circ} \mathrm{C}$ for further analysis.

UV treatment was given by exposing plants under UV C $\left(253 \mu \mathrm{mol} \mathrm{m} \mathrm{m}^{-2} \mathrm{~s}^{-1}\right)$ lamp for two minutes. Methyl jasmonate treatment was given by painting the leaves of C. roseus with Methyl jasmonate $(50 \mu \mathrm{M})$ using a cotton ball dipped in methyl jasmonate solution.

\section{Isolation of $\mathrm{CrMPK3}$}

A partial cDNA clone of $C$. roseus MAPK (438 bp) was amplified using the specific primers, MAPKF1: 5' -CTCAAGCTTCTTCGTCATAT-3' (forward) and MAPKR1: 5'-GACAGACCACACATCAATTG-3' (reverse) designed from CrMAPK EST (GenBank accession No. AJ537469). Amplification was carried out at $94^{\circ} \mathrm{C}$ for $30 \mathrm{sec}, 52^{\circ} \mathrm{C}$ for $30 \mathrm{sec}$, and $72^{\circ} \mathrm{C}$ for $30 \mathrm{sec}$ for 30 cycles using Taq DNA polymerase (Bangalore Genei, India). The partial CrMAPK was used as a probe to screen a $\lambda$-ZapII oriented YE elicited $C$. roseus leaf-specific cDNA library to get full length clone. Recombinant plaques were screened by plaque hybridization method. Positive plaque obtained after tertiary screening led to identification of a $630 \mathrm{bp}$ long partial CrMAPK clone with missing $5^{\prime}$ end. The $5^{\prime}$ end of the gene was successfully amplified using a combination of $5^{\prime}$ degenerate primer and reverse specific primer using $C$. roseus cDNA as template. The primer sequences were as follows: 5 '-ATGGYTGATGC WAATATGGGTG-3' (forward) and 5'-TTATGCA TATTCTGGATTTAGAGCC-3' (reverse). The amplified fragment was cloned into pGEM-T easy cloning vector (Promega), sequenced. Full length $C r M A P K$ clone was identified using ORF finder tool (NCBI). The full length cDNA of $C$. roseus MAPK was PCR amplified from C. roseus CDNA using gene specific primer pair (FLMPKF1: 5'-ATGGTTGATGCAAATATGG-3' and FLMPKR1: 5'TTATGCATATTCTGGATT-3') and cloned in PGEM-T Easy vector (Promega) and sequenced.

\section{Preparation of GST fusion proteins}

Full length CrMPK3 was cloned in expression vector pGEX-4 T-2 at BamHI and XhoI restriction sites. GSTCrMPK3 fusion protein was induced with $1 \mathrm{mM}$ isopropyl thio- $\beta$-D-galactosidase (IPTG) in E. coli strain BL-21. The fusion protein was purified using glutathione beads (GE Healthcare) following manufacturer's instructions.

\section{Generation of Kinase inactive GST-CrMPK3 fusion protein}

Kinase inactive GST:CrMPK3 fusion protein was generated by mutating a conserved lysine $(K)$ at $69^{\text {th }}$ residue in the ATP-binding domain of CrMPK3 to arginine (R). The directed mutagenesis was carried out using
CrMPK3 in pGEX-4 T-2 as template by mutagenic primer pair (5' -ATGGTGGCGATTAGGAAAATAGCT-3' and 5'-AGCTATTTTCCTAATCGCCACCAT-3'). The PCR amplification was carried out at $94^{\circ} \mathrm{C}$ for $30 \mathrm{sec}$, $52^{\circ} \mathrm{C}$ for $30 \mathrm{sec}$, and $72^{\circ} \mathrm{C}$ for $5 \mathrm{~min}$ for 30 cycles followed by final extension at $72^{\circ} \mathrm{C}$ for $10 \mathrm{~min}$. PCR product was digested overnight with $D p n \mathrm{I}$ and the digested product was transformed in protease deficient E. coli strain BL-21 (DE3). The point mutation K69R was verified by sequencing. The purified GST:CrMPK $3^{\mathrm{K} 69 \mathrm{R}}$ was obtained following the same protocol as mentioned before.

\section{In-gel kinase assay}

The in-gel kinase activity was carried out as described previously [26]. $20 \mu \mathrm{g}$ of total protein was fractionated on a $10 \%$ polyacrylamide gel containing $0.1 \%$ SDS and $0.5 \mathrm{mg} / \mathrm{ml}$ bovine brain myelin basic protein (MBP) (Sigma Aldrich). After electrophoresis, the SDS from the gel was removed with buffer $(25 \mathrm{mM}$ Tris- $\mathrm{HCl} \mathrm{pH} \mathrm{7.5,}$ $0.5 \mathrm{mM}$ DTT, $5 \mathrm{mM} \mathrm{Na}_{3} \mathrm{VO}_{4}, 0.1 \mathrm{mM} \mathrm{NaF}, 0.5 \mathrm{mg} \mathrm{ml}^{-1}$ $\mathrm{BSA}, 0.1 \%$ Triton $\mathrm{X} 100$ ) followed by renaturation in buffer $(25 \mathrm{mM}$ Tris-HCl pH 7.5, $0.5 \mathrm{mM}$ DTT, $5 \mathrm{mM}$ $\mathrm{Na}_{3} \mathrm{VO}_{4}, 0.1 \mathrm{mM} \mathrm{NaF}$ ) at $4^{\circ} \mathrm{C}$ overnight. MBP phosphorylation was performed by incubating the gel in $20 \mathrm{ml}$ of reaction buffer (25 mM Tris $\mathrm{HCl} \mathrm{pH} 7.5,2$ mM EGTA, $12 \mathrm{mM} \mathrm{MgCl} 2,1 \mathrm{mM}$ DTT, $0.1 \mathrm{mM} \mathrm{Na}_{3} \mathrm{VO}_{4}, 1 \mu \mathrm{M}$ ATP and $50 \mu \mathrm{Ci}$ of $\gamma 32 \mathrm{P}$-ATP $\left(3000 \mathrm{Ci} \mathrm{mmol}{ }^{-1}\right)$ for $60 \mathrm{~min}$ at room temperature. The gel was washed three times with 5\%TCA and 1\%Sodium pyrophosphate and dried on filter paper and autoradiographed in phosphorimager (Typhoon, GE health care).

\section{In solution kinase assay}

GST fusion protein $(5 \mu \mathrm{g})$ was incubated with $5 \mu \mathrm{g}$ of MBP and $1 \mu \mathrm{Ci}$ of $\gamma^{-}{ }^{32} \mathrm{P}$-labelled ATP in a $15 \mu$ reaction mixture (25 mM Tris-Cl pH 7.5, $5 \mathrm{mM} \mathrm{MgCl}_{2}, 25 \mu \mathrm{M}$ ATP, $1 \mathrm{mM}$ EGTA and $1 \mathrm{mM} \mathrm{DTT}$ ) at $30^{\circ} \mathrm{C}$ for $20 \mathrm{~min}$ utes. The reaction was stopped with $5 \times$ SDS loading buffer and the samples were resolved on a $15 \%$ polyacrylamide gel. The gel was dried on Whatman 3MM paper and exposed to X-ray film.

\section{Protein extraction and immunoblot analysis}

Frozen leaf tissue was ground in liquid nitrogen and homogenized in $1 \mathrm{ml}$ of ice-cold extraction buffer (100 mM HEPES -KOH pH 7.5, 5 mM EDTA, 5 mM EGTA, $10 \mathrm{mM}$ DTT, $10 \mathrm{mM} \mathrm{Na} \mathrm{VO}_{4}, 10 \mathrm{mM} \mathrm{NaF}$, $50 \mathrm{mM} \beta$-glycerol phosphate, $1 \mathrm{mM}$ PMSF, $10 \%$ glycerol and $7.5 \%$ PVPP). Extracts were centrifuged and the clear supernatant was recovered. For immunoprecipitation $200 \mu \mathrm{g}$ of extracted crude protein was incubated with $1 \mu \mathrm{g}$ of $4 \mathrm{G10}$ antiphosphotyrosine monoclonal antibody (Upstate Biotechnology, NY)/ dually phosphorylated 
active form of the MAPK ERK1, pTEpY antibody (Cell Signalling Technology) in $250 \mu \mathrm{l}$ volume of extraction buffer (without PVPP) with $250 \mathrm{mM} \mathrm{NaCl}$ and $0.1 \%$ (v/v) Nonidet P40. The assay was shaken at $4^{\circ} \mathrm{C}$ for $2 \mathrm{~h}$ and after the subsequent addition of $30 \mu \mathrm{l}$ of protein A-Sepharose (GE Healthcare) for overnight, followed by centrifugation $(13,000 \mathrm{rpm})$ for $3 \mathrm{~min}$ at $4^{\circ} \mathrm{C}$. The beads were washed thrice with extraction buffer, and boiled for $5 \mathrm{~min}$ in $30 \mu \mathrm{l}$ of $5 \mathrm{x}$ loading buffer $(0.625 \mathrm{M}$ Tris $-\mathrm{HCl}$ pH 6.8, 5\%SDS, 50\%glycerol, 0.125\%bromophenol blue and DTT). The reaction products were used for protein gel blot analysis as described previously [38]. CrMPK3 polyclonal antibody was custom synthesized from Gene Care, India. Briefly, purified recombinant CrMPK3 was provided for raising polyclonal antibody in rabbit. The preimmune serum and serum after inoculation were collected and tested for their ability to bind the $C$. roseus proteins in immumoblot analysis. The preimmune serum failed to detect any protein band specific to $C$. roseus in immunoblot analysis (data not shown) A primary antibody dilution of 1:2000 was used for anti-CrMPK3.

\section{Generation of construct for sub-cellular localization study} and transient transformation of $C$. roseus

Full length $C r M P K 3$ and $C r M P K 3^{K 69 R}$ was amplified with primers containing $N c o$ I and SpeI restriction sites in the forward and reverse primers respectively. For subcellular localization construct, reverse primer lacked the stop codon so that coding region of $C r M P K 3$ is in frame with reading frame of green fluorescent protein however for transient transformation construct stop codon was included in reverse primer. Respective amplicons were cloned in pGEM-T Easy vector and confirmed by sequencing. The constructs were generated by digesting CrMPK3 in pGEMT Easy vector with NcoI/ SpeI and inserting the excised fragment into the corresponding sites of the plant binary vector pCAMBIA 1303 digested with same set of restriction enzymes. The constructs were further confirmed by sequencing.

\section{RNA extraction and Northern Blot Analysis}

RNA extraction and Northern blot analysis were performed as described earlier [39]. $20 \mu \mathrm{g}$ RNA samples were separated on $1.2 \%$ denaturing agarose gel and blotted onto Hybond-N 14 membrane (GE, Piscataway, NJ, USA) using standard procedures. Prehybridization and hybridization of membranes were carried out at $60^{\circ} \mathrm{C}$ in modified church buffer (7\%SDS, 0.5 M NaPO4, $10 \mathrm{mM}$ EDTA, $\mathrm{pH}$ 7.2). Blots were hybridized with $\alpha-{ }^{32} \mathrm{P}-\mathrm{dCTP}-$ labeled full length cDNA clone of CrMPK3. Blots were washed in $2 \mathrm{XSSC}$ and $0.1 \%(\mathrm{w} / \mathrm{v})$ SDS at room temperature for $10 \mathrm{~min}$ followed by washing in $0.5 \mathrm{X}$ SSC and $0.1 \%(\mathrm{w} / \mathrm{v})$ SDS wash buffer at $60^{\circ} \mathrm{C}$ for $10 \mathrm{~min}$.
Membranes were exposed to Kodak X-OMAT film and signals were analyzed.

\section{Quantitative real time PCR (qRT-PCR) analysis}

Total RNA was extracted from the infiltrated leaves by $\mathrm{LiCl}$ method [39] and treated with 10 units of RNase free DNase 1 (Takara Bio Inc., Japan) to remove genomic DNA contamination. Five micrograms of total RNA was subjected to first strand synthesis using Power Script Reverse Transcriptase according to the manufacturer's procedure using oligo (dT) primer. qRT-PCR was conducted as have been described previously [20,40]. PCR amplification was conducted on one-tenth of the reaction. Primer pairs used for qRT-PCR analysis of different genes is mentioned in Additional file 8: Table 1.

\section{Transient transformation of $C$. roseus leaves by Vacuum infiltration}

CrMPK3/CrMPK $3^{K 69 R}$ in binary construct were used for transformation of competent cells of Agrobacterium tumefaciens strain GV3101. Six young leaves of C. roseus were submerged into a suspension of recombinant A. tumefaciens $\left(\mathrm{OD}_{600}=2\right)$ in MMA $(0.43 \%[\mathrm{w} / \mathrm{v}]$ Murashige and Skoog basic salt mixture [Sigma, Deisenhofen, Germany], $10 \mathrm{mM}$ MES [pH 5.6], 2\%[w/v] sucrose, $200 \mu \mathrm{M}$ acetosyringone) and incubated for $30 \mathrm{~min}$ under negative pressure generated by a vacuum pump. Leaves infiltrated with a suspension of empty vector were used as negative controls. Following infiltration, the leaves were briefly washed in tap water and dried on paper tissues to remove excess liquid. The leaves were placed on wet Whatman paper in plastic trays, sealed, and incubated for $60 \mathrm{~h}$ in a phytochamber at $25^{\circ} \mathrm{C}$, with a $16 \mathrm{~h}$ photoperiod.

\section{Total alkaloid extraction and HPLC analysis}

Alkaloid extraction and HPLC analysis was performed as mentioned elsewhere [34]. Briefly, total alkaloids were extracted from the dried leaf samples following the protocol described earlier [41]. Chromatographic separation of major indole alkaloids of $C$. roseus was carried out on a reversed-phase $\mathrm{C} 18$ column with binary gradient mobile phase profile [42]. Identification of the compounds were based on the retention time and comparison of the UV spectra with those of the authentic standards. Solutions of different authentic samples (catharanthine, vindoline, vinblastine, and serpentine) were prepared in methanol $\left(0.25 \mathrm{mg} \mathrm{ml}^{-1}\right)$ and used at different concentrations for the preparation of calibration graphs, linear in the range of $0.25-25 \mu \mathrm{g}$. Quantification analysis was repeated for three replicates of each tissue in parallel, and the means and standard deviations were calculated. 


\section{Additional files}

Additional file 1: Activation of MAP Kinases by MeJA treatment in C. roseus. 6-8 weeks old C. roseus plants were subjected to MeJA treatment at the mentioned concentrations. Control plant was given the mock treatment (Ethanol) in the same fashion as that of MeJA treatment. Activity of MAP kinase was assessed by in-gel kinase assay using MBP as a substrate polymerized in SDS 10\%(w/v) polyacrylamide gel.

Additional file 2: Phylogenetic relationship of CrMPK3 to other plant MAPKs. The amino acid sequence of the proteins used for generating phylogenetic tree is listed in GenBank database under the following accession numbers: OsMPK3 (A2XFC8), OsMPK6 (ABO69383), NtF4 (X83880), SIMK (X66469), PsD5 (X70703), AtMPK6 (D21842), ZmMPK5 (AB016802), WIPK (D61377), AtMPK3 (D21839), ZmMPK4 (AB016801), AsMAP1 (X79993), LeMPK3 (AAP20421) and StMAPK (BAE44363).

Additional file 3: Deduced amino acid sequence of CrMPK3. Amino acid sequence of CrMPK3 highlighting different subdomains of MAP kinase. Conserved TEY motif present between subdomain VII and VIII is underlined.

Additional file 4: Plasmolysis of $C$. roseus leaf cells to confirm the subcellular localization of CrMPK3-GFP fusion protein. Plasmolysis was achieved by incubating the cells in $1 \mathrm{M}$ mannitol solution for 2 hours.

Additional file 5: Nuclear localization of CrMPK3-GFP upon wounding. C. roseus leaf discs transiently transformed with CrMPK3-GFP cloned in PCAMBIA1303 vector and observed under confocal microscope 10 min after wounding. A-B: CrMPK3-GFP fluorescence and bright field respectively. C: DAPI staining shows position of nucleus.

Additional file 6: CrMPK3 activation in response to wounding, UV, MeJA treatments. Total protein extracts $(200 \mu \mathrm{g})$ was subjected to immunoprecipitation with the PTEPY antibody. The immunoprecipitated complex was electrophoresed on SDS 10\%(w/v) polyacrylamide gel and immunoblot was performed using anti-AtMPK3. MeJA mock treatment was performed by applying solvent only (ethanol) in the similar fasion to that of MeJA. Representative CBB stained total protein show equal loading control.

Additional file 7: Specificity of CrMPK3 antibody. Immunoblot was performed using CrMPK3 antibody against total protein extract of Arabidopsis Atmpk3 mutant line and wild type plants (Col 0).

Additional file 8: List of genes and primer pairs for Q RT-PCR.

\section{Abbreviations}

GFP: Green fluorescent protein; MeJA: Methyl jasmonate; MAPK: Mitogen activated protein kinase; MAPKK: Mitogen activated protein kinase kinase; MAPKKK: Mitogen activated protein kinase kinase kinase; MBP: Mylein basic protein; MIA: Monoterpenoid indole alkaloid; UV: Ultraviolet.

\section{Competing interests}

The authors declare that they have no competing interest.

\section{Authors' contributions}

SKR performed most of the experiments and drafted the manuscript, DPW conducted IP and in-gel kinase assays and helped in drafting the manuscript, MJ and SKJ helped in quantitative real time PCR (qRT-PCR) analysis, PS helped in constructs preparations, confocal microscopy and raising Arabidopsis mutant plants, AHS helped in confocal microscopy work, BR helped in western blot analysis. AKS conceived the idea, designed and coordinated the experiments and drafted the manuscript. All authors read and approved the final manuscript.

\section{Acknowledgements}

SKR, MJ, AHS, PS acknowledge Council of Scientific and Industrial Research, India, DPW thanks University Grant Commission, while BR and SKJ thank Department of Biotechnology, India for the fellowship. This work is supported by core grant of National Institute of Plant Genome Research, New Delhi, India from Department of Biotechnology, India.
Received: 28 May 2012 Accepted: 30 July 2012

Published: 7 August 2012

\section{References}

1. El-Sayed M, Verpoorte R: Catharanthus terpenoid indole alkaloids: biosynthesis and regulation. Phytochem Rev 2007, 6:277-305.

2. Aerts R, Gisi D, De Carolis E, De Luca V, Baumann TW: Methyl jasmonate vapor increases the developmentally controlled synthesis of alkaloids in Catharanthus and Cinchona seedlings. Plant J 1994, 5:635-643.

3. Menke FLH, Stefanie P, Mueller MJ, Kijne JW, Memelink J: Involvement of the Octadecanoid pathway and protein phosphorylation in fungal elicitor induced expression of Terpenoid Indole Alkaloid biosynthetic genes in Catharanthus roseus. Plant Physiol 1999, 119:1289-1296.

4. Thoma I, Loeffler C, Sinha AK, Gupta M, Krischke M, Steffan B, Roitsch T, Mueller MJ: Cyclopentenone isoprostanes induced by reactive oxygen species trigger defense gene activation and phytoalexin accumulation in plants. Plant J 2003, 34:363-375.

5. Ren D, Liu Y, Yang KY, Han L, Mao G, Glazebrook J, Zhang S: A fungal responsive MAPK cascade regulates phytoalexin biosynthesis in Arabidopsis. Proc Natl Acad Sci USA 2008, 105:5638-5643.

6. Sinha AK, Jaggi M, Raghuram B, Tuteja N: Mitogen-activated protein kinase signalling in plants under abiotic stress. Plant Signal Behav 2011, 6:196-203.

7. Suarez-Rodriguez MC, Petersen M, Mundy J: Mitogen-Activated Protein Kinase signalling in plants. Annu Rev Plant Biol 2010, 61:621-649.

8. Mishra NS, Tuteja R, Tuteja N: Signaling through MAP kinase networks in plants. Arch Biochem Biophys 2006, 452:55-68.

9. Taj G, Agarwal P, Grant M, Kumar A: MAPK machineryin plants: Recognition and response to different stresses through multiple signal transduction pathways. Plant Signal Behav 2010, 5:1370-1378.

10. Fiil BK, Petersen K, Petersen M, Mundy J: Gene regulation by MAP kinase cascades. Curr Opin Plant Biol 2009, 12:615-621.

11. Vazquez-Flota FA, De Luca V: Jasmonate modulates development and light regulated alkaloid biosynthesis in Catharanthus roseus. Phytochemistry 1998, 49:395-402.

12. Ouwerkerk PBF, Hallard D, Verpoorte R, Memelink J: Identification of UV-B light responsive regions in the promoter of tryptophan decarboxylase gene from Catharanthus roseus. Plant Mol Biol 1999, 41:491-503.

13. Vazquez-Flota FA, Carrillo-Pech M, Minero-Garcia Y, Miranda-Ham ML: Alkaloid metabolism in wounded Catharanthus seedlings. Plant Physiol Biochem 2004, 42:623-628.

14. Hamel LP, Nicole MC, Sritubtim S, Morency MJ, Ellis M, Ehlting J, Beaudoin N, Barbazuk B, Klessig D, Lee J, Martin G, Mundy J, Ohashi Y, Scheel D, Sheen J, Xing T, Zhang S, Seguin A, Ellis BE: Ancient signals: comparative genomics of plant MAPK and MAPKK gene families. Trends Plant Sci 2006, 11:192-198.

15. Hyun TK, Hoffmann A, Sinha AK, Roitsch T: Tomato mitogen activated protein kinases regulate the expression of extracellular invertase Lin6 in response to stress related stimuli. Funct Plant Biol 2009, 36:1088-1097.

16. Ligterink W, Kroj T, zur Nieden U, Hirt H, Scheel D: Receptor-mediated activation of a MAP kinase in pathogen defense of plants. Science 1997, 276:2054-2057.

17. Seo S, Sano H, Ohashi Y: Jasmonate based wound signal transduction requires activation of WIPK, a tobacco mitogen activated protein kinase. Plant Cell 1999, 11:289-298.

18. Holley SR, Yalamanchili RD, Moura DS, Ryan CA, Stratmann JW: Convergence of signaling pathways induced by systemin, oligosaccharide elicitors, and UV-B radiation at the level of mitogen activated protein kinases in Lycopersicon peruvianum suspensioncultured cells. Plant Physiol 2003, 132:1728-1738.

19. Ahlfors R, Macioszek V, Rudd J, Brosché M, Schlichting R, Scheel D, Kangasjärvi J: Stress hormone-independent activation and nuclear translocation of mitogen-activated protein kinases in Arabidopsis thaliana during ozone exposure. Plant J 2004, 40:512-522.

20. Jaggi M, Kumar S, Sinha AK: Overexpression of an apoplastic peroxidase gene CrPrx in transgenic hairy root lines of Catharanthus roseus. Appl Microbiol Biotech 2011, 90:1005-1016.

21. Liu Y, Zhang S: Phosphorylation of 1-aminocyclopropane-1-carboxylic acid synthase by MPK6, a stress-responsive mitogen-activated protein 
kinase, induces ethylene biosynthesis in Arabidopsis. Plant Cell 2004, 16:3386-3399.

22. Miles GP, Samuel MA, Zhang Y, Ellis BE: RNA interference-based (RNAi) suppression of AtMPK6, an Arabidopsis mitogen-activated protein kinase, results in hypersensitivity to ozone and misregulation of AtMPK3. Environ Pollut 2005, 138:230-237.

23. Zhang S, Liu Y, Klessig DF: Multiple levels of tobacco WIPK activation during the induction of cell death by fungal elicitins. Plant J 2000 23:339-347.

24. Liu Y, Jin H, Yang KY, Kim CY, Baker B, Zhang S: Interaction between two mitogen-activated protein kinases during tobacco defense signaling. Plant J 2003, 34:149-160

25. Sinha AK, Hofmann MG, Römer U, Köckenberger W, Elling L, Roitsch T: Metabolizable and non-metabolizable sugars activate different signal transduction pathways in tomato. Plant Physiol 2002, 28:1480-1489.

26. Rao KP, Vani G, Kumar K, Sinha AK: Rhythmic expression of Mitogen Activated Protein Kinase activity in rice. Mol Cells 2009, 28:417-422.

27. Ramani S, Chelliah J: UV-B-induced signaling events leading to enhanced production of catharanthine in Catharanthus roseus cell suspension cultures. BMC Plant Biol 2007, 7:61-77.

28. Dutta A, Singh D, Kumar S, Sen J: Transcript profiling of terpenoid indole alkaloid pathway genes and regulators reveals strong expression of repressors in Catharanthus roseus cell cultures. Plant Cell Rep 2007, 26:907-915.

29. Moreno-Valenzuela OA, Galaz-Avalos RM, Minero-garcia Y, Loyola-Vargas VM: Effect of differentiation on the regulation of indole alkaloid production in Catharanthus roseus hairy roots. Plant Cell Rep 1998, 18:99-104.

30. Vazquez-Flota F, De Luca V, Carrillo-Pech M, Canto-Flick A, de Lourdes Miranda-Ham M: Vindoline biosynthesis is transcriptionally blocked in Catharanthus roseus cell suspension cultures. Mol Biotechnol 2002, 22:1-8.

31. Kurusu T, Hamada J, Nokajima H, Kitagawa Y, Kiyoduka M, Takahashi A, Hanamata S, Ohno R, Hayashi T, Okada K, Koga J, Hirochika H, Yamane H, Kuchitsu K: Regulation of microbe-associated molecular pattern-induced hypersensitive cell death, phytoalexin production, and defense gene expression by calcineurin B-like protein-interacting protein kinases, OsCIPK14/15, in rice cultured cells. Plant Physiol 2010, 53:678-692.

32. Pauw B, Hilliou FAO, Sandonis Martin V, Chatel G, de Wolf CJF, Champion A, Pre M, Van Duijn B, Kijne JW, van der Fits L, Memelink J: Zinc finger proteins act as transcriptional repressors of alkaloid biosynthesis genes in Catharanthus roseus. J Biol Chem 2004, 279:52940-52948.

33. van der Fits L, Memelink J: ORCA3, a jasmonate-responsive transcriptional regulator of plant primary and secondary metabolism. Science 2000 289:295-297.

34. Dutta A, Batra J, Pandey-Rai S, Singh D, Kumar S, Sen J: Expression of terpenoid indole alkaloid biosynthetic pathway genes corresponds to accumulation of related alkaloids in Catharanthus roseus (L.) G. Don. Planta 2005, 220:376-383.

35. Xu J, Li Y, Wang Y, Liu H, Lei L, Yang H, Liu G, Ren D: Activation of MAP kinase kinase 9 induces ethylene and camalexin biosynthesis and enhances sensitivity to salt stress in Arabidopsis. J Biol Chem 2008, 283:26996-27006.

36. Kishi-Kaboshi M, Okada K, Kurimoto L, Murakami S, Umezawa T, Shibuya N, Yamane H, Miyao A, Takatsuji H, Takahashi A, Hirochika H: A rice fungal MAMP responsive MAPK cascade regulates metabolic flow to antimicrobial metabolite synthesis. Plant J 2010, 63:599-612.

37. Sottomayor M, Cardoso IL, Pereira LG, Ros Barceló A: Peroxidase and the biosynthesis of terpenoid indole alkaloids in the medicinal plant Catharanthus roseus (L.) G. Don. Phytochem Rev 2004, 3:159-171.

38. Samuel MA, Miles GP, Ellis BE: Ozone treatment rapidly activates MAP kinase signalling in plants. Plant J 2000, 22:367-376

39. Kumar S, Dutta A, Sinha AK, Sen J: Cloning, characterization and localization of a novel basic peroxidase gene from Catharanthus roseus. FEBS J 2007, 274:1290-1303.

40. Kumar K, Rao KP, Sharma P, Sinha AK: Differential regulation of rice mitogen activated protein kinase kinase (MKK) by abiotic stress. Plant Physiol Biochem 2008, 46:891-897.

41. Singh DV, Maithy A, Verma RK, Gupta MM, Kumar S: Simultaneous determination of Catharanthus alkaloids using reversed phase high performance liquid chromatography. J Liq Chromatogr 2000, 23:601-607.
42. Tikhomiroff C, Jolicoeur M: Screening of Catharanthus roseus secondary metabolites by high-performance liquid chromatography. J Chromatogr 2002, 955:87-93.

doi:10.1186/1471-2229-12-134

Cite this article as: Raina et al.: CrMPK3, a mitogen activated protein kinase from Catharanthus roseus and its possible role in stress induced biosynthesis of monoterpenoid indole alkaloids. BMC Plant Biology 2012 12:134.

\section{Submit your next manuscript to BioMed Central and take full advantage of:}

- Convenient online submission

- Thorough peer review

- No space constraints or color figure charges

- Immediate publication on acceptance

- Inclusion in PubMed, CAS, Scopus and Google Scholar

- Research which is freely available for redistribution
Ciomed Central 